\begin{tabular}{|c|c|}
\hline 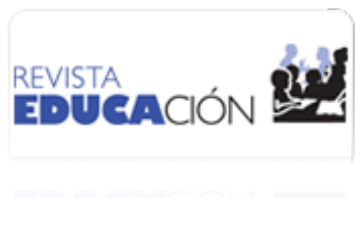 & $\begin{array}{l}\text { Revista Educación } \\
\text { ISSN: 0379-7082 } \\
\text { ISSN: } 2215-2644 \\
\text { revedu@gmail.com } \\
\text { Universidad de Costa Rica } \\
\text { Costa Rica }\end{array}$ \\
\hline
\end{tabular}

\title{
Alfabetización mediática en América Latina. Revisión de literatura: temas y experiencias
}

\author{
Garro-Rojas, Lidieth \\ Alfabetización mediática en América Latina. Revisión de literatura: temas y experiencias \\ Revista Educación, vol. 44, núm. 1, 2020 \\ Universidad de Costa Rica, Costa Rica \\ Disponible en: http://www.redalyc.org/articulo.oa?id=44060092042 \\ DOI: https://doi.org/10.15517/revedu.v44i1.37708
}

Esta obra está bajo una Licencia Creative Commons Atribución-NoComercial-SinDerivar 3.0 Internacional 


\title{
Alfabetización mediática en América Latina. Revisión de literatura: temas y experiencias
}

\author{
Media Literacy in Latin America: Issues and Experiences \\ Lidieth Garro-Rojas \\ Universidad de Costa Rica, Costa Rica \\ DOI: https://doi.org/10.15517/revedu.v44i1.37708 \\ Redalyc: http://www.redalyc.org/articulo.oa?id=44060092042
}

lidieth.garro@ucr.ac.cr

(iD http://orcid.org/0000-0003-3822-8468

Recepción: 29 Julio 2019

Aprobación: 17 Diciembre 2019

\section{Resumen:}

Se analiza la literatura académica producida en Latinoamérica referida a las experiencias de alfabetización mediática con el fin de examinar la importancia del establecimiento de iniciativas de alfabetización mediática en esta región. Se desarrolla una amplia revisión bibliográfica y entre los hallazgos se encuentra que las principales discusiones en el subcontinente giran en torno a iniciativas de alfabetización mediática orientadas a la comprensión de contenidos y lenguajes, seguido del uso de herramientas tecnológicas y posteriormente el análisis de las condiciones institucionales en las cuales se desarrolla, o no, la alfabetización mediática. En la mayoría de los países latinoamericanos el surgimiento del campo de la alfabetización mediática se da más como resultado de las iniciativas de organizaciones y de algunas personas académicas interesadas en el tema, quienes generan reflexión sobre estas experiencias como producto de una agenda académica o de política educativa de interés para las autoridades educativas. Palabras Clave: Comunicación y educación, Alfabetización mediática, Educación sobre medios de comunicación, América Latina.

\section{AbSTRACT:}

Latin American academic literature on Media Literacy was examined to determine the importance of establishing Media Education initiatives in this region. After conducting a broad literature review, it was determined that the main discussion points in the subcontinent revolve around media literacy aimed at understanding content and language, followed by the use of technology and examination of institutional conditions that are conducive, or not, to media literacy. In most Latin American countries, the emergence of Media Literacy has been the result of organizational initiatives and scattered academic interest in this topic with the goal of provoking thought about experiences resulting from academic or political educational agendas of interest to academic authorities.

KEYWORDS: Communication and Education, Media Literacy, Media Education, Latin America.

\section{INTRODUCCIÓN}

En el mundo de hoy los medios de comunicación digitales y tradicionales son una fuente de información/ formación y entretenimiento en constante movimiento, generando nuevos códigos, espacios de complicidad y construcciones cognitivas (Garro y Martínez, 2013). La omnipresencia de diversos medios de comunicación en la vida cotidiana sugiere la vastedad del campo y que la educación para la lectura audiovisual del mundo que nos rodea es uno de los retos de nuestro tiempo.

Sin embargo, las discusiones sobre la alfabetización mediática o la relación de la educación con el uso de los medios de comunicación es un campo que apenas da sus primeros pasos en el mundo académico de América Latina. A pesar de contar con una larga historia en países como Francia, Inglaterra y Canadá, la aparición en el ámbito académico latinoamericano no solo es más reciente, sino que adquiere una intensidad menor en cuanto a cantidad de publicaciones y discusión académica relacionada. 
En este artículo se entiende la alfabetización mediática como la adquisición de capacidades para interpretar y transformar códigos del sistema mediático de radiodifusión sonora y televisiva, así como los medios que hacen uso de las TIC y los medios impresos.

En el mundo académico, Alfabetización Mediática e Informacional es un concepto en debate que hace referencia a una serie de abordajes y prácticas los cuales abarcan desde la formación de las personas usuarias para que sean capaces de utilizar las tecnologías de la información y la comunicación hasta aquellas que proponen esta como un derecho que permitiría a la población contar con elementos para hacer juicios sobre la realidad mediática y social en la que ejercen la ciudadanía. Así, el concepto de alfabetización mediática es contextual e histórico y se encuentra en proceso de cambio, pues la aparición de nuevos soportes de información y comunicación, nuevos contextos culturales y sociopolíticos traen consigo nuevas estrategias de aprendizaje y formas de lectura.

Al tratarse de un campo emergente se hace necesario contar con una perspectiva reflexiva sobre cuáles son los principales temas de debate y aspectos prácticos reportados en la literatura académica del subcontinente el cual permita comprender los principales retos y vías de desarrollo que en esta región lo orientan. Así, este artículo busca responder a la pregunta ¿qué aspectos se problematizan en los debates sobre la alfabetización mediática en la literatura académica sobre América Latina?, para ello se examinan artículos académicos publicados entre los años 2011 y 2016 que reportan, sistematizan o analizan experiencias prácticas del campo de la alfabetización mediática en esta región.

Fedorov (2011) elabora un recorrido histórico de la educación en medios desde su nacimiento en la década de 1920, en Francia, hasta nuestros días y hace un balance de la situación en diversas regiones, sin embargo, omite criterio sobre América Latina. El estudio de los recorridos de la discusión, las experiencias y los debates en esta región son aún un tema pendiente.

Con este aporte se pretende arrojar luz a un campo del saber que desde el punto de vista disciplinar se configura en el intersticio de los debates sobre la educación, la comunicación y la participación ciudadana. Un campo con vida propia que como producto del desarrollo tecnológico se ve arrastrado y constreñido por los debates que el desarrollo de las tecnologías de la información y la comunicación, sus apropiaciones y los usos que generan.

Se trata de un campo que se instaura en la tensión entre lo propuesto por la UNESCO en diversos textos sobre lo que es y debe ser la alfabetización mediática e informacional y las propias reflexiones producto de la experiencia que se llevan a cabo en contextos y con acceso a recursos particulares.

A diferencia de lo que se ha instituido en otras latitudes (Fedorov, 2011), en la mayoría de los países de América Latina la educación para los medios ocupa un lugar marginal o del todo no forma parte del currículo académico en la escuela primaria ni en la secundaria. Ello produce un desarrollo menor de experiencias y de reflexiones que podrían generarse al calor de esa condición de institucionalización.

Se presenta un debate en ciernes y por ello resulta de utilidad recurrir a las nociones del campo de Bourdieu (1994), que recuerdan cómo la constitución de los campos académicos y disciplinares se dirimen a partir de relaciones de fuerza entre los agentes y las instituciones que intervienen en la lucha; la distribución del capital específico que ha sido acumulado en las luchas anteriores orienta las estrategias posteriores. Las luchas en el campo ponen en acción el monopolio de la violencia legítima (autoridad específica del campo) que es, en definitiva, la lucha por la conservación o la subversión de la redistribución de la legitimidad dentro de este.

En relación con la constitución del campo académico, Sánchez (2007) indica que este es un espacio complejo que incluye los roles de producción de conocimiento asumido por las personas académicas e investigadoras; la distribución que es asumida por docentes e instancias de difusión, tales como publicaciones científicas; el consumo, conformado por estudiantes, personas que investigan y, finalmente, las instancias de legitimación, tales como universidades e institutos de investigación. Desde esta perspectiva, en este artículo se centra la atención en los aspectos vinculados a la producción y distribución de conocimiento. 


\section{Alfabetización Mediática: CONCEPTO EN PROCESO}

Uno de los conceptos fundadores para la alfabetización mediática es el establecido por Aufderheide (1993) en la National Leadership Conference on Media Literacy que la definió como "the ability of a citizen to access, analyze, and produce information for specific outcomes." [es la capacidad del ciudadano para acceder, analizar y producir información para resultados específicos] (p. 6). Desde ese texto fundacional el concepto es objeto de diversas disputas, según la autora para algunos expertos decodificación explica mejor que análisis o evaluación; la producción se explica mejor como codificación; la información era interpretada como simples símbolos hasta las formas más complejas que incluían el mundo digital multimedia. La inclusión de la idea de resultados también era cuestionada en sus alcances para los sujetos.

Esta dificultad para establecer un concepto único de la alfabetización mediática da cuenta de la diversidad de prácticas comunicativas en la vida cotidiana, la educación, las relaciones interpersonales y los ejercicios de la vida pública propician el incesante desarrollo tecnológico y el dinamismo del campo teórico de la comunicación que tiene como reto el análisis que vincula los medios tradicionales y los más favorecidos con el desarrollo tecnológico con procesos sociales reticulares. En ese sentido, Garro y Martínez (2013) citando a Bernabéu, Esteban, Gallego y Rosales (2011), indican la dificultad de contar con un concepto único de alfabetización, pues este se encuentra en proceso de cambio debido a la aparición de nuevos soportes de información y comunicación que conllevan igualmente nuevas estrategias y formas de lectura. Igualmente, Potter (2013) indica la dificultad existente para definir a qué se refiere e indica que más que una definición única, existen tres núcleos temáticos alrededor de los cuales esta gira: ¿qué son los medios?, ¿a qué se refiere la alfabetización? y ¿cuál es el propósito de la alfabetización mediática? Las respuestas a estos aspectos producen una serie de definiciones que, según el Potter (2013):

almost all of the ideas presented complement one another, that is, there is little evidence of different groups of scholars
arguing about which group has the best definition. There seems to be a rather general agreement about some core ideas and a
general acceptance of a great many peripheral ideas, such that the differences across definitions seem to be traceable more to
what each scholar chooses to emphasize in a particular definition.[Casi todas las ideas presentadas se complementan entre
sí, es decir, hay poca evidencia de que diferentes grupos de académicos discutan sobre qué grupo tiene la mejor definición.
Parece haber un acuerdo bastante general sobre algunas ideas centrales y una aceptación general de una gran cantidad de
ideas periféricas, de modo que las diferencias entre las definiciones parecen más relacionadas con lo que se elige enfatizar en
una definición particular] (p.421).

El autor indica que las discusiones conceptuales se agrupan alrededor de siete aspectos centrales: los medios de comunicación (MC) tienen el potencial de ejercer una amplia gama de efectos sobre las personas; los MC ejercen influencia sobre los individuos pero también en las estructuras sociales; debido a que la influencia de los MC es constante y sutil, las personas son más susceptibles a esa influencia cuando son pasivas; el objetivo de la alfabetización mediática no es solo ayudar a las personas a protegerse de los efectos potencialmente negativos de los MC, sino también capacitarlas para su uso en función de sus propios objetivos; la alfabetización mediática debe ser desarrollada; es multimensional y, finalmente, implica la adquisición de conocimiento y el desarrollo de pensamiento crítico. (Potter, 2013).

Pérez (2015) se refiere a la alfabetización mediática e informacional como:

un nuevo paradigma conceptual referido a las capacidades que permiten el uso crítico, autónomo y creativo -por parte de las personas y las comunidades- de cualquier medio de información y comunicación y expresión y de sus lenguajes específicos. Estas capacidades van desde las habilidades técnicas más sencillas de uso de los medios hasta las cognitivas empleadas en el procesamiento de la información y en la utilización de los lenguajes y el razonamiento -mucho más complejas- (p. 4).

En ese sentido Wilson, Grizzle, Tuazon, Akyempong y Cheung, (2011) relacionan con la noción de alfabetización mediática una serie de habilidades y competencias que incluyen entender el papel y las funciones de los medios; las condiciones bajo las cuales estos pueden cumplir sus funciones en las sociedades democráticas; la evaluación crítica del contenido de estos a la luz de las funciones que tienen en las sociedades 
democráticas; el compromiso con la autoexpresión y la participación democrática y el desarrollo de destrezas, incluidas el manejo de TIC, necesarias para producir contenido. Estos aspectos vinculan la alfabetización mediática con la perspectiva de las políticas públicas en materia de educación y los derechos humanos y (UNESCO, 2008; Tornero y Varis, 2010; Aguaded, 2012) la necesidad de fomentar esta alfabetización como la forma de abordar una de las barreras que impiden el desarrollo personal y la incorporación a los procesos sociales, especialmente aquellos vinculados al ejercicio democrático. Desde el punto de la investigación en comunicación, da cuenta del tránsito del estudio de los modelos de los medios masivos de comunicación hacia las prácticas interactivas y en red en que los sujetos desarrollan sus vínculos sociales.

En perspectiva, el concepto de alfabetización debe ser situado en el contexto sociopolítico, cultural y tecnológico. Hoy día no basta contar con una alfabetización que otorgue competencias para decodificar el texto impreso y expresarse en esa forma de comunicación; ni siquiera es suficiente comprender otros lenguajes audiovisuales y sus interactividades y ser capaces de expresarse éticamente a través de ellos. También es necesario que el sujeto cuente con nociones mínimas que le permitan comprender aspectos básicos de la economía de los medios, las fragmentaciones culturales que el desarrollo tecnológico propicia y favorece y las necesarias interacciones existentes y por construir entre los contenidos mediáticos, el ejercicio de la ciudadanía y vivir una vida con calidad.

\section{Metodología de ANÁLISIS}

Para la elaboración de esta revisión se efectuó una amplia búsqueda de artículos en bases académicas tales como Proquest, Scielo, Scielo de Brasil, Unirioja y Ebsco, Google Académico y repositorios de diversas universidades de América Latina[1]. En total se revisaron más de 100 bases de datos de texto completo, dicha búsqueda permitió finalmente conformar un corpus de 26 artículos académicos publicados en castellano, portugués o inglés, que dan cuenta de reflexiones, experiencias y discusiones conceptuales latinoamericanas sobre la alfabetización mediática y que en fueron publicadas entre los años 2011 y 2016. La busca incluyó los descriptores de educomunicación, alfabetización mediática, educación en medios, educación para los medios.

Se desarrolló un proceso de análisis y codificación de los artículos, que en primera instancia llevó a la generación de códigos descriptivos, producto de la agrupación temática de los contenidos desarrollados en cada artículo.

Esta primera etapa de codificación dio paso a la creación de categorías analíticas que se desarrollan en este artículo.

Se contó con el apoyo de la herramienta de análisis cualitativo NVivo, la cual facilitó la sistematización de la literatura académica y la posterior organización del corpus de análisis en los cuatro ejes que corresponden a los aspectos principales del debate latinoamericano sobre alfabetización mediática y que se desarrolla a continuación.

Para esta segunda etapa fueron de utilidad las reflexiones de Peguer y Martínez (2016). Su trabajo establece tres ejes de estudio: dimensiones, actores y medios de comunicación. En cuanto a la primera, se incluyen las problemáticas de acceso y uso, la comprensión crítica y la producción de contenidos; el corpus mostró la necesidad de nuevas dimensiones de análisis, como el caso de las condiciones institucionales en las que se desarrolla la alfabetización mediática.

\section{Resultados}

Los artículos publicados hacen referencia a investigaciones, experiencias ya finalizadas o en desarrollo o propuestas que abarcan, según orden de importancia: comprensión de contenidos y lenguajes, el aprendizaje del uso de herramientas tecnológicas, el análisis de las condiciones institucionales que dificultan o facilitan 
la implementación o la profundización de la educación en medios. Además, dos temas adicionales: la experiencia en el uso de canales de comunicación para la implementación de proyectos de educación en medios y el análisis y la monitorización. Estos últimos responden a las condiciones específicas que disfrutó Argentina durante el período en que estuvo en vigencia la Ley de Servicios de Comunicación Audiovisual.

A continuación, se presenta un análisis más detallado de cada uno de estos aspectos.

\section{Comprensión de contenidos y lenguajes}

La presentación y explicación de iniciativas orientadas a la comprensión los lenguajes y análisis de contenidos es por mucho la temática que más se reitera en el corpus de análisis.

Trece de las personas autoras presentan reflexiones en este ámbito: Andrelo y Ferrari (2015), Gasca (2010), García y Rivera (2013), Hernando y Phillippi (2013), Marín (2010), Pérez (2009), Pérez y Sandoval (2013), Rodrigues (2014), Ruberti y Nascimento (2001), Salgado et al. (2012), Santos y Akhras (2011), Gonzálvez y Zeballos (2013) y Soriano (2013).

Dentro de esta amplia área temática destacan las reflexiones que vinculan los medios de comunicación, la comprensión y la lectura crítica como aspectos que favorecen el ejercicio de la ciudadanía desde aspectos como la lectura de los medios de comunicación en el ámbito de una campaña electoral, la participación de las personas en la definición de la agenda mediática, la incorporación de elementos de interpretación de alfabetización visual al currículo escolar y la interpretación de le mensajes publicitarios, entre otros.

En este sentido Andrelo y Ferrari (2015) muestran que las personas jóvenes consideran la exhortación a la memoria como una forma legítima de argumentar durante las campañas electorales y por tanto, la destacan como un aspecto a abordar en las acciones de alfabetización mediática. Destacan la importancia de incluir en la formación en medios la reflexión sobre el papel y las características de la memoria para un ejercicio consciente del derecho al voto. Ambos llegan a estas conclusiones a partir del análisis de una experiencia desarrollada en Brasil con jóvenes a quienes se les solicitó el análisis de propaganda electoral y trabajaran sobre la memoria y el papel que esta cumple en la argumentación de la propaganda electoral.

Sumado a la memoria, García y Rivera (2013) propone dotar a la ciudadanía con herramientas para el análisis de la definición de la agenda (Agenda Setting) en los medios de comunicación; esto como una vía para contribuir a la construcción ciudadana. En la perspectiva de la autora, esta competencia les permitiría comprender cómo los medios de comunicación definen qué es y qué no es importante de ser discutido por la opinión pública; además, comprender quién decide los temas que deben o no ser conocidos y discutidos. Propone la incorporación en la formación escolar de competencias transversales para el análisis de la agenda de los medios, de manera que se fortalezcan las capacidades para su análisis y asumir las decisiones de su uso de manera consciente y comprometida con los valores personales y el referente social. Ejecuta la propuesta a partir de un análisis de contenido de prensa escrita. Rodrigues (2014) propone, tanto en calidad de metodología como de tema, la incorporación de la comprensión de imágenes y de los nuevos medios en las clases de artes del sistema escolar formal; asimismo formar docentes que sean capaces de utilizarlas, analizarlas, discutirlas y hacerlas accesibles a sus estudiantes. El autor conjuga en su propuesta una visión política de análisis de las imágenes, los medios y los mensajes, que hace referencia a la necesidad de fortalecer las capacidades para efectuar lectura crítica de medios. En ese mismo sentido en el análisis de Pérez (2009), la comprensión de los contenidos se orienta a fortalecer las capacidades de lectura crítica, que sustente el ejercicio delpensamiento libre y critico de los mensajes mediáticos; se trata de fortalecer la capacidad de razonamiento de la lectura y el pensamiento crítico y autónomo, así como las competencias que permitan al sujeto entender, analizar e interpretar lo que lee, y también la clara expresión de opiniones. Nuevamente, se vinculan estas capacidades como propias de un ejercicio ciudadano autónomo y reflexivo.

En relación con los sujetos que deben formarse para la comprensión de textos de medios de comunicación, una parte importante de la literatura revisada establece implícita o explícitamente, que se trata de personas 
jóvenes y el espacio de aprendizaje es la educación formal, tal como lo explicitan, entre otros, Pérez (2009) y Rodrigues (2014). Sin embargo, una excepción lo constituye el aporte de Hernando y Phillippi (2013), para quienes uno de los retos de la sociedad de la información en su conjunto es dotar a distintos sujetos, y entre ellos a las personas mayores, de herramientas para la comprensión de los medios de comunicación.

En cuanto a los medios propios para ese ejercicio ciudadano, los aportes hacen referencia a mensajes provenientes de la televisión (Andrelo y Ferrari, 2015), Pérez y Sandoval (2013), Rodrigues (2014); la prensa escrita (Pérez, 2009), Pérez y Sandoval (2013) y los medios electrónicos (Gasca, 2010). Sobresale, como ausencia, un medio clásico como la radio.

En relación con las habilidades de lectura crítica existentes, Gasca (2010) toma en consideración la competencia electrónica, la lectura en internet, las características de un lector crítico y las competencias que requiere el alumnado para desarrollar la literacidad crítica; llega a la conclusión de que los jóvenes mexicanos, objeto de su investigación, adquieren capacidades especialmente en lo relativo a la búsqueda y recuperación de textos en internet. Soriano (2013) sugiere que la alfabetización en la virtualidad debe advertir las implicaciones del consumo de medios, los procesos socioculturales y la construcción de significados.

Destaca en este apartado que, en varios de los artículos analizados, al hablar de la necesidad de fortalecer las competencias para el uso del lenguaje se hace referencia implícita a textos escritos, sin referencias claras al uso de las imágenes audiovisuales o los textos multilenguajes e interactivos. Como excepciones destacan los aportes de Santos y Akhras, (2011) y Marín (2010), quienes hacen un llamado a reforzar la lectura y comprensión de otros lenguajes, como el cinematográfico, las animaciones, las infografías y las telenovelas. Cabe desatacar que únicamente el texto de Santos y Akhras (2011) se refiere a la formación en lenguaje cinematográfico y exposición a películas del cine.

En una discusión totalmente aparte, más propia de la tradición norteamericana, Salgado et al. (2012) desarrolla un estudio en el cual demuestran que existe una relación cercana entre la exposición de jóvenes a mensajes publicitarios y la incidencia del fumado y, por otra parte, altas capacidades de comprensión de contenidos y lectura crítica con una incidencia menor en el fumado actual y la susceptibilidad en el fumado futuro. Siempre en relación con las preocupaciones por la relación de las personas jóvenes con la publicidad, Pierre (2012) hace un llamado para que se respeten las franjas horarias infantiles en las televisoras pautando ahí únicamente contenidos publicitarios adecuados para los menores de edad.

En síntesis. En la literatura académica analizada destaca la preocupación de formar, de manera prioritaria a las personas jóvenes en la comprensión del lenguaje y varios autores vinculan estas competencias con las necesarias para el ejercicio de la ciudadanía.

Aunque no en todos los artículos queda claro que al hablar de lenguajes se hace referencia a algo que no sea lenguaje escrito, también se incorporan preocupaciones por el fortalecimiento de la lectura de las imágenes, especialmente las vinculadas con la televisión y en menor medida la reflexión sobre la virtualidad y el uso de internet. Cabe destacar la ausencia de los mensajes radiofónicos en general como parte de los textos analizados.

También se desarrolla, en menor medida, la preocupación por el fortalecimiento de las capacidades de lectura crítica. Esta toma forma en aspectos como la comprensión de los mecanismos de la definición de la agenda mediática (Agenda Setting) y la comprensión de la memoria para el análisis de mensajes.

Exceptuando uno de los artículos, que desarrolla la necesidad de brindar formación específica a personas mayores en el uso de Internet, destaca la ausencia a cualquier referencia a las brechas etarias o de acceso a los medios de comunicación.

\section{Alfabetización como uso de herramientas TIC}

El uso de herramientas, particularmente TIC, es el tema que ocupa el segundo lugar entre los más presentes en el grupo de artículos analizados. 
Se razona que las herramientas tecnológicas contribuyen a que las personas puedan desarrollar la creatividad y la productividad; que sean usuarias informadas, responsables y capaces de contribuir a su entorno inmediato y como sociedad y que puedan colaborar, publicar y producir conocimiento. Su potencialidad se encuentra en que pueden favorecer el desarrollo personal y social de los sujetos (Cisneros, 2010; Hernando y Phillipi, 2013; Ruberti y Nascimento, 2001).

En particular en el ámbito de la educación formal, Cárdenas y Vargas (2010) y Cabello (2011) proponen contextualizar las competencias y conocimientos TIC que se requieren, tanto en términos de las necesidades sociales como de los recursos de que se disponen.

Cabello (2011), citando a Esperón (2003), indica que es necesaria una pedagogía la cual dé cuenta de la ampliación de los espacios y los tiempos de aprendizaje y los procesos de relación y comunicación, que estimule la creación y el descubrimiento de redes que consideren los medios tecnológicos y humanos. Cabello (2011) resume: es necesario promover la discusión y la intervención sobre qué tipo de sujetos se requiere formar, y en relación con ello, docentes con competencias en cuáles áreas para que asuman el reto de orientar esos procesos de formación en los contextos específicos; ello implica la identificación de requerimientos de formación en el uso de las tecnologías que estas sirvan a la tarea de potenciar los procesos y prácticas educativas.

Si bien es cierto prácticamente todos los autores que problematizan sobre la necesidad de fortalecer el uso de las tecnologías (Cárdenas y Vargas, 2010; Cabello, 2011; Cisneros, 2010; Rodrigues, 2014; Ruberti y Nascimento, 2001 y Soriano, 2013) expresan la necesidad de vincular su aprendizaje al currículo escolar o en el establecimiento de programas de formación para docentes, cabe señalar el aporte de Hernando y Phillippi (2013) quienes ponen en discusión las particularidades que implica promover el uso de las TIC entre las personas adultas mayores.

Consideran Hernando y Phillippi (2013) que cuando se trata de personas mayores no se debe hablar del manejo de herramientas, sino de las utilidades prácticas para la vida en las que las tecnologías pueden mejorar la calidad de vida: pedir una cita médica, ejecutar gestiones bancarias o institucionales, localizar a un antiguo familiar o llamar a un amigo. Con esta población se debe atender la resistencia al cambio y los miedos que el uso de los dispositivos puede provocar entre estas personas.

\section{Condiciones institucionales para la alfabetización mediática}

El tercer aspecto destacado se refiere al análisis que hacen las personas autoras de las condiciones institucionales en las que se efectúa la alfabetización mediática. Peguer y Martínez (2016) afirman que en Latinoamérica las iniciativas de alfabetización mediática están frecuentemente ligadas a la educación no formal, la educación popular y la sociedad civil. Estos autores, citando a Aguaded (1995) y Fantin (2011), destacan que a menudo se trata de iniciativas que se originaron en el contexto sociopolítico de la oposición a las dictaduras militares y que en su nacimiento orientaron su quehacer a fortalecer en la población las herramientas para la lectura crítica del cine y los medios de comunicación.

En las circunstancias actuales, se estaría hablando de alfabetización mediática en un contexto en el que, al menos formalmente, la mayoría de los países de la región gozan de democracia expresada como alternabilidad en el poder y en muchos de ellos libertad de prensa.[2]

Los avances en las condiciones institucionales en los que se desarrolla la alfabetización mediática son relativamente pocos y diferenciados entre países. La literatura revisada permite una mirada general en algunos de ellos:

Argentina: Existencia de una Defensoría del Público capaz de atender y canalizar los reclamos de las audiencias y producir materiales de capacitación (Cortés, Bacher y Romero, 2016); existió, durante el período de la presidenta Fernández un canal infantil (Pakapaka) y en diversos programas (Notipakapaka, Los secretos 
de la animación, El Show de Perico) se tematizó la producción de los medios de comunicación (Sánchez, 2013).

Brasil: Los artículos revisados dan cuenta de la existencia de una amplia variedad de proyectos orientados a la alfabetización mediática Andrelo y Ferrari, (2015); Santos y Akhras, (2011), llegando Peguer y Martínez (2016) a estimar en 240 los proyectos e iniciativas provenientes de la sociedad civil en el 2016. No obstante, García, Matos y Borges (2016) afirmaban que en este país la alfabetización mediática continúa sin ser incluida en los currículos escolares, a pesar de las discusiones y las presiones al Ministerio de Educación.

Colombia: El plan de Educación (aprobado para el período 2006-2015) reconocía la influencia de los medios de comunicación en la educación infantil; sin embargo, Pérez y Sandoval (2013) indican que la educación en medios, más que producto de la planificación institucional, es producto de la iniciativa de educadores con pasión por el tema que han liderado proyectos que constituyen una forma inicial de integración entre el currículo y la educación en medios.

El Salvador: El currículo aprobado en el 2008, indica Soriano (2013), incluye un apartado denominado Informática, en su opinión el más cercano a lo que podría considerarse alfabetización mediática. Centrado en el uso de las TIC, y sin tomar en consideración el ecosistema mediático, se propone el desarrollo de cuatro competencias: investigación e interpretación de información; producción y comunicación rápida y efectiva; resolución de problemas utilizando tecnologías y utilización ética y eficiente de las TIC.

México: Se carece de una propuesta de alfabetización establecida desde el currículo y el tema no adquiere relevancia en la agenda pública (Cortés et al, 2016). Tejedor y Esquivel (2011) señalan la importancia de que la alfabetización ciberperiodística forme parte del currículo periodístico, ausente aún en el caso mexicano.

Lo que Cortés et al, (2016) indican para México tiene validez para casi todo el contexto regional. Aunque en algunas universidades y centros educativos el profesorado y las y los investigadores han brindado aportaciones al estudio y han desarrollado experiencias, se carece de planes suficientemente consistentes para lograr su difusión y aplicación a nivel nacional o incorporar el tema de forma decidida al currículo escolar. Igualmente, el debate, cuando se presenta, sigue siendo propio de especialistas y no alcanza relevancia en la agenda pública.

Como advertencia necesaria, no se pretende indicar que únicamente existen en la región las iniciativas sobre las que dan cuenta los artículos analizados, pero sí que la alfabetización mediática, como problema de discusión académica y como campo de conocimiento, tiene una extensión relativamente reducida. En ese sentido, contrario a lo que podría implicar un avance en la democratización de las sociedades latinoamericanas, el vínculo entre democracia, educación para los medios o alfabetización mediática y educación ha desarrollado una discusión escasa en el ámbito académico.

\section{Procesos de producción de contenidos}

Uno de los aspectos centrales para la alfabetización mediática es la apropiación de los procesos de producción de contenidos por parte de los sujetos usuarios de los medios de comunicación. Así lo indica buena parte de la literatura. Sin embargo, únicamente dos de las experiencias reportadas en la literatura analizada, el Proyecto Bem-te-vi y Un minuto por mis derechos reportan centrar su actividad en brindar a niños, niñas y jóvenes conocimientos y experiencia que les facilita la elaboración de audiovisuales.

El proyecto brasileño Bem te vi (Costa, 2010) no solamente reporta la elaboración de más de 130 videos sino también el desarrollo de metodologías diferenciadas para cada uno de los grupos atendidos según condición económica, manejo de la lengua portuguesa, zona geográfica, entre otras particularidades.

Por otra parte, el proyecto Un minuto por mis derechos (Caturegli, 2011), proyecto argentino con el apoyo de UNICEF, promovía la expresión de las personas jóvenes sobre sus derechos a través de videos de un minuto de duración. Se conjuga la reflexión acerca de los derechos con el fortalecimiento de herramientas para que asuman la palabra, su manera de contar, desde la producción audiovisual. 


\section{Conclusiones}

La búsqueda efectuada en bases de datos permitió la construcción de un corpus el cual permite el análisis en profundidad de la producción académica en América Latina sobre alfabetización mediática.

Una primera constatación es que este es un tema marginal en la agenda académica latinoamericana en términos de cantidad de producción y de discusión entre autores. La cantidad es escasa y no se presenta un verdadero diálogo entre abordajes metodológicos, perspectivas teóricas o resultados de experiencias. Así, los textos analizados permiten dar cuenta de un campo del saber que se construye a lo largo del subcontinente a partir de temáticas pertinentes, pero diversas y dispersas; sobresalen preocupaciones como el aprendizaje de los lenguajes, los usos de tecnologías, la lectura crítica y el análisis de las condiciones institucionales para el desarrollo de la alfabetización mediática.

Discursivamente un eje que articula varias de producciones académicas es la preocupación por vincular las prácticas y reflexiones sobre la alfabetización mediática con el ejercicio de la ciudadanía a través y desde los medios de comunicación. A ello suman aspectos aquí analizados como la capacidad de lectura crítica de medios, que se vincula al ejercicio ciudadano reflexivo y autónomo.

Si se puede hacer un boceto de los sujetos que son tema de preocupación de la literatura analizada diremos que se trata, en general, de jóvenes que se encuentran vinculados al sistema educativo formal. Únicamente en uno de los artículos se presenta una propuesta para abordar el tema con adultos mayores. Sin embargo, son notables las ausencias de otras poblaciones que pueden tener necesidades específicas, tales como mujeres y personas indígenas.

Cabe destacar que esto coloca en perspectiva la aseveración de Peguer y Martínez en el sentido de que la mayoría de las experiencias latinoamericanas de alfabetización mediática son lideradas por organizaciones de la sociedad civil. Ello propone el reto de reconstruir las experiencias lideradas de sectores diferentes al estatal, pues la experiencia acumulada en cada uno de los países no está quedando como memoria sistematizada en el campo académico.

Contradictoriamente, aunque se reconoce que la mayoría de las experiencias de alfabetización son lideradas desde la sociedad civil, muchos de los textos argumentan sobre la importancia de incluir estos temas en el currículo escolar y de las universidades, mientras se pone en evidencia las grandes ausencias en ambos espacios educativos. Poco se reporta de las experiencias que se presentan al margen del sistema educativo.

Con el fin de contar con un panorama más certero de cómo se construye el campo académico de la alfabetización mediática en la región, es necesario contar con más sistematizaciones de aquellas experiencias que organizaciones de la sociedad civil y populares han venido desarrollando para acercar los medios a la gente. Se sabe, por ejemplo, en el caso de Costa Rica, que son múltiples las iniciativas existentes, sin embargo, la experiencia acumulada no ha sido sistematizada y no es posible encontrar referencia a ellas en la literatura académica.

La academia podría tener un papel preponderante en la recuperación de las experiencias lideradas desde el mundo de las organizaciones privadas. Con toda seguridad se ha generado ahí un saber hacer que sería de gran utilidad para procesos de institucionalización de la alfabetización mediática.

Uno de los aspectos que contribuye de manera más directa a la vinculación de la alfabetización mediática con el ejercicio de la ciudadanía es el fortalecimiento de las capacidades de producción para diferentes medios de comunicación. Esa es, precisamente, una de las temáticas que se encuentra representada en menor medida dentro de los textos analizados. Adicionalmente, la mención a las capacidades de lectura y producción de un medio tradicional y tan extendido como la radio, se encuentra ausente.

Las grandes fragmentaciones en las acciones y en el debate sobre la alfabetización mediática urgen a la necesidad de profundizar en el reconocimiento de las condiciones institucionales en las que esta actividad se desarrolla. Es válido preguntar para cada país cuál es la disposición y la apertura de los ministerios de educación para incluir este tema en la formación escolar de jóvenes e infantes. Reviste particular interés 
el conocimiento de cómo se incorpora al currículo escolar esta formación; en este sentido los estudios comparativos y el análisis de experiencias específicas permitirían la profundización de la formación a partir de experiencias en curso.

Finalmente, la perspectiva de trabajo supranacional permitiría avanzar en la instauración del tema a paso firme, reconociendo los aciertos y errores cometidos en otros países latinoamericanos y favoreciendo el aporte que desde este ámbito de trabajo se puede hacer al desarrollo de la democracia.

\section{REFERENCIAS}

Aguaded, I. (1995). La educación para la comunicación: la enseñanza de los medios en el ámbito hispanoamericano. En I. Aguaded y J. Cabero (Ed.), Educación y medios de comunicación en el contexto iberoamericano. Seminario Internacional sobre Educación y medios de comunicación en el contexto Iberoamericano (pp. 19-48). Huelva: Universidad Internacional de Andalucía, La Rábida.

Aguaded J. I. (2012). Media proficiency, an educational initiative that cannot wait. Comunicar, 20(1), 07-09. doi: h ttps://doi.org/10.3916/C39-2012-01-01

Andrelo, R. y Ferrari, W. (2015). Media literacy, memória e eleições - como jovens interpretam o apelo à memória na campanha presidencial de 2014. Comunicação \& educação, 20(2), 27-36.

Aufderheide, P. (1993). Media Literacy: A Report of the National Leadership Conference on Media Literacy. Maryland, United States: Aspen Institute, Communications and Society Program. Recuperado de https://files.eric.ed.go v/fulltext/ED365294.pdf

Bernabéu, N., Esteban, N., Gallego, L., Rosales, A. (2011).. España: Ministerio de educación. Secretaria General Técnica. Subdirección General de Documentación y Publicaciones. Recuperado de https://sede.educacion.gob.es/publiven ta/PdfServlet?pdf=VP15157.pdf\&area=E

Bourdieu, P. (1994). El campo cientifico. Redes: revista de estudios sociales de la ciencia, 1(2), 129-160. Recuperado de http://ridaa.unq.edu.ar/handle/20.500.11807/317

Cabello, R. (2011). Tecnovector. Migraciones digitales como propuesta de alfabetización mediática digital en la formación docente. En R. Cabello y S. Morales (Eds.) Enseñar con tecnologias. Nuevas miradas en la formación docente (pp.17-46). Buenos Aires: Prometeo.

Cárdenas, N. y Vargas, M. (2010). Alfabetización en TIC: un proceso de investigación con profesores de instituciones oficiales en Girardot - Cundinamarca- Colombia. En J.M. Pérez (Presidencia). Congreso Euro-Iberoamericano de Alfabetización Mediática y Culturas Digitales, Sevilla, España.

Caturegli, R. (2010) Lenguaje audiovisual, educación y derechos. En J.M. Pérez (Presidencia). Congreso EuroIberoamericano de Alfabetización Mediática y Culturas Digitales, Sevilla, España.

Cisneros, N.G. (2010). Uso de medios alternativos como estrategia de promoción y desarrollo de la alfabetización mediática. En J.M. Pérez (Presidencia). Congreso Euro-Iberoamericano de Alfabetización Mediática y Culturas Digitales, Sevilla, España.

Cortés, J.A., Bacher, S. y Romero, L.M. (2016). Alfabetización mediática en la formación del profesorado y comunicadores. The Journal of Media Literacy, 63(1 - 2), 93-100.

Costa, A. (2010). Bem-te-vi: a produção audiovisual de crianças e adolescentes. En J.M. Pérez (Presidencia). Congreso Euro-Iberoamericano de Alfabetización Mediática y Culturas Digitales, Sevilla, España.

Esperón, T. (2003). La comunicación y la educación, una alianza necesaria en la escuela. En J.I. Aguaded (Coord). Luces en el laberinto audiovisual. (pp. 83-93) España: Universidad de Huelva, Grupo de investigación Agora, Grupo de Investigación Comunicar.

Fantin, M. (2011). Mídia-educacão: aspectos históricos e teórico- metodológicos. Olhar de Professor, 14(1), 27-40. doi: http://doi.org/10.5212/olharprofr.v.14i1.0002

Fedorov, A. (2011). Breve repaso histórico. Alfabetización mediática en el mundo. Revista Infoamérica. (5), 7-23. 
García, R. y Rivera, D. (2013). Competencia mediática ante la Agenda Setting: parámetros de actuación a nivel educativo. Chasqui Revista Latinoamericana de Comunicación, (124), 48-54. doi: https://doi.org/10.16921/ch asqui.v0i124.18

García, R., Matos, A y Borges, G. (2016). Media Literacy as a responsability of families and teachers. The Journal of Media Literacy, 63(1 - 2), 82-91.

Garro, L. y Martínez, Y. (2013). Alfabetización mediática: reconociendo brechas, desarrollando estrategias y aprendiendo lectura crítica. Experiencias de formación en alfabetización mediática y lectura crítica con docentes de escuelas primarias de la provincia de San José. En S. Fridman y R. Edel Navarro (Eds). Ciencias, tecnologias y culturas: Educación y nuevas tecnologias. (pp. 325-333) México: Red de Integración Latinoamericana en Educación y Tecnología.

Gasca, M.A. (2010). Desarrollo de la literacidad crítica en Internet en estudiantes mexicanos de Bachillerato. En J.M. Pérez (Presidencia). Congreso Euro-Iberoamericano de Alfabetización Mediática y Culturas Digitales, Sevilla, España.

Gonzálvez, V. y Zeballos, R. (2013). Las interacciones mediáticas de la juventud boliviana: la competencia mediática de jóvenes de La Paz. Chasqui Revista Latinoamericana de Comunicación, (124), 34-40.

Hernando, A. y Phillipi, A. (2013). El desarrollo de la competencia mediática en personas mayores: una brecha pendiente. Chasqui Revista Latinoamericana de Comunicación, (124), 11-18.

Marín, B.E. (2010). De alfabetizaciones y multialfabetizaciones en la infografía digital. En J.M. Pérez (Presidencia). Congreso Euro-Iberoamericano de Alfabetización Mediática y Culturas Digitales, Sevilla, España.

Peguer, M. y Martínez J.F. (2016). Media Literacy in Brazil: Experiences and Models in Non-formal Education. Comunicar, 49, 39-48. doi: https://doi.org/10.3916/C49-2016-04.

Pérez, A. (2009). El desarrollo de la lectura crítica: una vía hacia la alfabetización mediática en educación superior. Revista Letras, 51(79), 309-356.

Pérez,J. M. (2015). La emergencia de la alfabetización mediática e informacional Un nuevo paradigma para las políticas públicas y la investigación. Revista TELOS (Cuadernos de Comunicación e Innovación), 100, 99-102.

Pérez, A. y Sandoval, Y. (2013). Avances para el desarrollo de la competencia mediática a partir del currículum de primaria en Colombia y España. Chasqui Revista Latinoamericana de Comunicación, (124), 26-33.

Pierre, K. (2012). Niñez, adolescencia, publicidad y alfabetización mediática. Revista Reflexiones, 90(1), 125-131.

Potter, W. J. (2013). Review of literature on media literacy. Sociology Compass, 7(6), 417-435. doi: https://doi.org/ $10.1111 /$ soc 4.12041

Rodrigues, W. (2014). Letramento imagético e midiático em arte-educação. Revista conhecimento e diversidade (12), 90-101. doi: http://dx.doi.org/10.18316/1607

Ruberti, I. y Nascimento A. (2001). Mídia, educação e cidadania: consideraçöes sobre a importância da alfabetização tecnológica audiovisual na sociedade da informação. Educação Temática digital Campinas, 3(1), 21-27.

Salgado, V., Pérez, E., Kaplan, P., Alderete, E., Mejía, R., Primack, B. y Gregorich, S. (2012). Association of Media Literacy with Cigarette Smoking Among Youth in Jujuy, Argentina. Nicotine \& Tobacco Research, 14(5), $516-$ 521

Sánchez, J y Méndiz, H. (2013). La alfabetización mediática en la televisión infantil online: programas del Canal Pakapaka. Chasqui Revista Latinoamericana de Comunicación, (124), 56-62.

Sánchez, R. A. (2007). La teoría de los campos de Bourdieu, como esquema teórico de análisis del proceso de graduación en posgrado. Revista electrónica de investigación educativa, 9(1), 1-21. Recuperado de https://bit.ly/2Ybb4l

Santos, L. y Akhras, F. (2011). Media Literacy: uma experiência brasileira. Rumores, 5(10), 80-104. doi: https://doi. org/10.11606/issn.1982-677X.rum.2011.51253

Soriano, A.M. (2013). Educación mediática 2.0. Revista Diá-logos. 7(12), 17-43. doi: https://doi.org/10.5377/dialo gos.v0i12.2192 
Lidieth Garro-Rojas. Alfabetización mediática en América Latina. Revisión de literatura: temas y e...

Tejedor, S. y Esquivel, F. (2010). De la alfabetización mediática a la alfabetización ciberperiodística: Un estudio de caso: Olimpiadas para Jóvenes Ciberperiodistas de México. En J.M. Pérez (Presidencia). Congreso EuroIberoamericano de Alfabetización Mediática y Culturas Digitales, Sevilla, España.

Tornero, J. M. P. y Varis, T. (2010). Media Literacy and New Humanism. Russian Federation: UNESCO. Recuperado de https://iite.unesco.org/pics/publications/en/files/3214678.pdf

UNESCO. (2008). Indicadores de desarrollo mediático: marco para evaluar el desarrollo de los medios de comunicación social. Paris: UNESCO

Wilson, C., Grizzle, A., Tuazon, R., Akyempong, K. y Cheung, Ch. (2011). Alfabetización mediática e informacional. Curriculum para profesores. Paris: UNESCO.

\section{Notas}

[1] Una base de información bibliográfica elaborada por la autora, con publicaciones sobre alfabetización mediática e informacional en inglés, portugués y español se encuentra en: http://www.kerwa.ucr.ac.cr/handle/10669/73478

[2] La Relatoría Especial para la Libertad de Expresión adscrita a la Organización de Estados Americanos (OEA) (http:// www.oas.org/es/cidh/expresion/index.asp) cuenta con información detallada sobre las condiciones para el ejercicio de la libertad de expresión en América Latina.

\section{BY-NC-ND}

